\title{
Metasystem approach to increase the load factor FMS
}

\author{
Alexandr Pishchukhin ${ }^{1, *}$ \\ ${ }^{1}$ Orenburg state university, Orenburg, Russia
}

\begin{abstract}
The work is devoted to increasing the efficiency of using flexible manufacture systems (FMS) by increasing the load factor. Unlike the well-known multi-agent approach to production management, we are investigating the addition of a metasystem level allowing flexible combination of the decentralized control method with centralization. The metasystem approach allows to reduce management to flexible switching of functioning technologies on the basis of the replacement rule, while describing FMS as a metasystem consisting of classification of the system analyst J. Klir from structured data systems. Production events are simulated in virtual space with the help of a multi-agent approach. At the same time, the number of requests from the waiting queue is chosen such that it becomes possible to optimize the loading of the FMS in the simplest case by the full-scan method, if the queue is not available, then the initiative of choice is provided to communications of agents-technologies and agents-equipments. Experimental studies were carried out on the FMS model including six pieces of equipment divided into three groups with a stream of six applications, each of which has a technological route of six operations each.. The experimental results showed the consistency of the developed approach by increasing the load factor of the FMS.
\end{abstract}

\section{Introduction}

Universal flexible manufacture system (FMS) has in its arsenal a significant set of production equipment. And although in its operation they try to apply the method of group technology [1], there is equipment that does not participate in the production process, which is bad for the load factor (on the ratio of the employment time to the total running time of the FMS). This problem is solved by compiling an optimal schedule [2] with strict centralization of its implementation, but with a sufficiently large load, many of its variants appear and it is difficult to select an optimization method that ensures sufficient speed. On the other hand, with increasing complexity, you can move to decentralized management and use the simulation method of modeling the production environment [3-5], such as agent-oriented programming. However, this eliminates the possibility of optimization where it is possible. Let us examine the question of the combination of centralization and decentralization through timely switchovers. A metasystem approach to management, developed from the beginning of the year 2000 , can serve as a theoretical basis for solving switching issues and their timeliness [6].

\section{Theory}

In accordance with J. Klir's systemology [7], there are two ways of integrating systems: creating a structured system and creating a metasystem (See Figure 1). Each system is described by a set of variables, the changes of which are considered on a set of parameters. In structured systems, integration is performed over sets of variables under the assumption that they all have the same parametric set. Thus, the elements of structured systems are systems with different sets of variables, but with the same parametric sets. In metasystems, on the contrary, systems are integrated over parametric sets, regardless of whether these systems have one set of variables or not.
Structured system

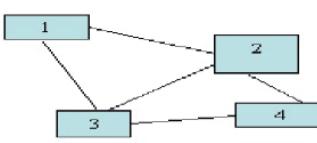

Metasystem

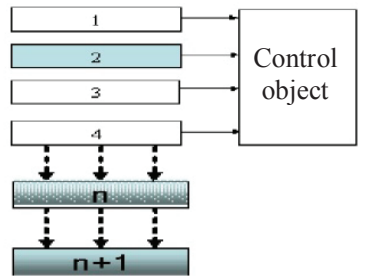

Fig. 1. System Integration Scheme.

As a consequence of this definition, the difference between the two ways of integrating systems, also in that the structured system reacts to external influences by changing the magnitudes of the variables in the relationships between the elements, the metasystem (which acts as a slider) can, in addition, replace the functioning system, that is reacts more drastically. In addition, in a structured system, all the elements function simultaneously, so if any one element fails, the entire system ceases to function. In a metasystem, a functioning system is selected by the replacement rule and, upon failure, can be replaced by another capable of functioning.

\footnotetext{
* Corresponding author: pishchukhin55@mail.ru
} 
For these reasons, the metasystem has a higher survivability a priori. By survivability, it should be understood as a longer working capacity due to better adaptability [8].

The metasystem is defined as a triple:

$$
\mathbf{M S}=(\mathbf{W}, S, r)
$$

where $\mathbf{W}$ is a parametric set; $S$ - can be a set of any systems, in this case it is a set of structured systems whose parametric sets are subsets of $\mathbf{W} ; r$ is a replacement procedure realizing a certain function of the form

$$
r: \mathbf{W} \rightarrow S
$$

This formula defines the replacement function.

Metasystems are divided into two large classes [6]: sequential and parallel actions, so the replacement function for the second class selects a group of simultaneously functioning systems. For the most effective implementation of the replacement of one functioning system by another, it is necessary to set and solve six metasystem tasks [9]: identification of subbands for the efficient functioning of systems; assessment and improvement of the required level of system availability for use; development of a switching strategy for individual or groups of simultaneously functioning systems; identifying and ensuring the compatibility, coherent interaction of systems; optimal redistribution of limited system-wide resources; optimal synthesis of the metasystem.

\section{Data and methods}

Let us now look at the FMS and present it in the form of a metasystem that receives the flow of service requests, as was done in [10], consisting of structured systems, which in turn consist of data systems

$$
\mathbf{M S D}=(\mathbf{W}, S D, r)
$$

With the rule of replacement of the technological process

$$
r: \mathbf{W} \rightarrow S D
$$

A lot of parameters in this case $\mathbf{W}$ will be represented by an assortment of manufactured products. For example, for a machining FMS, this can be: product housings, shafts, gears, levers, bushings and so on. Then the set $S D$ represents the set of technological processes for manufacturing the above assortment, since the structured systems themselves are integrated as follows from the above theory, we will consider them to be components of structured systems-data systems. Each of these data systems includes a description of the operations of the manufacturing process (cutting modes, types of equipment, tools, devices). Thus, each of the technological processes includes a set of interrelated operations (structure). For example, $S D_{i}=\left\{\left({ }^{x} V,{ }^{x} D\right) \mid\right.$ $\left.x \in N_{q}\right\}$, where ${ }^{x} V$ is the set of binding variables for the data system $x,{ }^{x} D$ is the set of data systems, $q$ is the number of elements in the structured system, and $N$ is the number of data systems in the $j$ - th element of a structured system.

In the case of FMS, the structured system will simulate a technology that includes associated data systems for the mastering equipment, the product design with surface treatment methods, and technological processing modes. Metasystem accordingly will be at the top level of management, in its charge will be all resources of FMS in the form of technological, transport and storage equipment

In addition, the solution of the six above-mentioned tasks of the metasystem approach will allow to structure the optimization of the production process in the FMS. When solving the first problem, it is important to remember that the metasystem arises there and then, when and where the range of the problem being solved is so great that it is not blocked by the functioning of one system, or this system does not function effectively in some parts of the general range. Therefore, the problem arises of identifying the boundaries separating the subranges of the effective functioning of systems. For example, in a FMS one and the same surface can be processed by different technologies. These subbands can be identified using a multidimensional feature space.

The second problem is related to the fact that there is a period of time in which unused systems stand idle and, therefore, lose their readiness for use. For technologies included in the FMS, the loss of readiness is associated with equipment malfunction, lack of blanks, raw materials, materials used for this technology. Readiness can be assessed by the probability that the system will function at the time of switching to it. It is possible to control this probability only by creating favorable conditions for the appearance of the desired event, or eliminating the reasons for its non-appearance.

The third task of the metasystem approach is prognostic and is linked to the FMS with the production schedule $[11,12]$.

The fourth task involves some interconnection of systems, for example at the structural level, when combinations of some systems in operation give a greater effect than their other combinations. For example, the functioning of a technology for manufacturing a product on the market and the technology using the resulting waste, or the functioning of two related technologies with a dramatically changing demand for each type of products manufactured with their help - the "emissions" of the market can be skillfully smoothed, flexibly managing the release volumes.

The fifth task arises only when using the group technology method in FMS, while the need for their coordination, optimization of resources directed to each system, to achieve the most effective metasystem, comes to the fore. At the same time, system-wide resources are directed to a greater extent in the system that most increases the metasystem effect.

Finally, the sixth task is connected with the optimization of the set of systems in the metasystem. As a criterion, one can choose the specific effect coefficient, that is, the ratio of the effect of including this technology in the metasystem portfolio to the costs associated with its acquisition and development, and then systems with the 
largest specific effect coefficient are included in the portfolio first. This task, primarily related to optimizing the set of equipment in the FMS

When planning the loading of FMS, which is represented as a metasystem, it is necessary to select the replacement rule in such a way that the load factor of the equipment is the maximum. And in this case, a certain generalized load factor is required because it must take into account the loading of many pieces of equipment. It can be taken in the form:

$$
K_{z}=\frac{\sum_{1}^{N} k_{i}}{N \cdot T}
$$

where $N$ is the number of pieces of equipment, $T$ is the total running time of the FMS, $k_{i}$ is the load factor of the $i$-th equipment unit.

\section{Methods or model}

In this paper, we use an agent-based model for planning a loading strategy for equipment, as having several advantages [13-15]:

- a continuous stream of events at the entrance allows the system to automatically react to changes in the state of orders and resources in real time;

- every order and every resource (in this case equipment) of the enterprise receives its program agent in the virtual space, which is triggered by the events that occur, to improve its current performance;

- Agents of each order and resource build their own, but connected in a common network of schedules, in such a network the schedule can contain hundreds of thousands of interrelated transactions;

- the basis for the approach to planning is not a complete or partial combinatorial search of options, but the identification and resolution of conflicts through negotiation of agents and the achievement of compromises - just as people do in real life;

- the created plan is launched for execution, during which the metasystem monitors the execution and initiates re-planning in case of discrepancies between the plan and the fact.

Multi-agent systems are a promising direction of simulation modeling, considered as independent from around 1990, when Job Shochem [16] formulated the main concepts of this new paradigm for solving complex problems.

However, this approach means fully decentralized management, in which the global minimum of managerial costs for the result can be missed. It is proposed to apply a multi-agent (agent-oriented) approach, in which there are active agent-technologies in the virtual space, whose purpose is to continuously assess the level of their readiness for launch and a metasystem that monitors the continuity of the production process and replaces the functioning technology as the production resources are freed. The metasystem in the regular case is guided by a previously developed technology switching strategy. Agents-technologies also prepare and launch their technology in accordance with this strategy, as well as in the case of an order from the metasystem.
The implementation of the described model was carried out with the help of an appropriate computer program. For simplicity, only standard switching for six technologies was simulated in it. At the same time, the usual multi-agent management of FMS, in which the preparation and launch of technology into production began in a decentralized manner, was compared with multi-agent, in which the metasystem first developed an optimal strategy in the virtual space and combined it in management with the production environment.

Each agent-technology monitors the freed machines and for operations that are not implemented in their technological map. If such conditions are found, it raises the "flag". The metasystem follows the equipment and does not allow downtime of any machine. As soon as the operation on any machine ends, a technology agent with a raised "flag" is searched. So the metasystem functions in the normal mode when the flow of orders arrives. If orders are known in advance, it tries to simulate in the virtual space communication agents-equipment and technology agents. With a small number of orders, as in the case considered in this paper, with six machines and six technologies with six operations, each has 720 variants of permutations, so the task can be optimized by the method of full search.

\section{Results}

The results are shown in Figure 2, where different colors depict functioning technologies and their switching from one equipment to another. On the vertical axis are six types of equipment that implement these technologies the absence of color on a horizontal line means simple equipment. The horizontal axis represents time. As you can see, the figure shows an ordinary example of a production schedule with a load factor of 0.83 . Preliminary optimization of the production process in the virtual space by the method of full enumeration allows you to bring the load factor to 0.917 .

\section{Conclusions or discussion and implication}

Thus, the metasystem approach allows us to concentrate the researchers' attention on optimizing the process at the strategic level, freeing up routine operational management, giving freedom to decentralized management using active agents in the virtual space at the tactical level. The mechanism of metasystem 


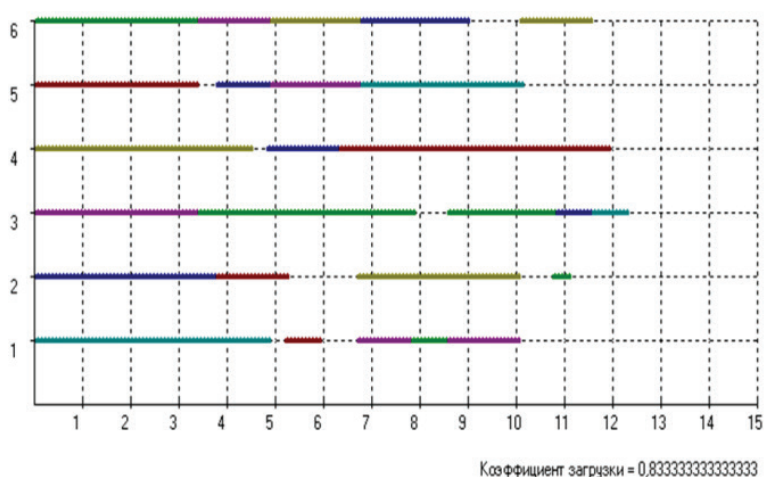

Vertical 1-6 - equipment number, horizontally - time

Fig. 2. Scheme of technology switching by equipment groups.

switchings, while providing apparatus for describing FMS as metasystems, consisting of structured systems that describe the technologies that appear in orders. The obtained experimental results allow to draw a conclusion about the operability and advantage of the agent-oriented method, combined with the metasystem approach to managing the FMS with respect to increasing its load factor

Further studies may be related to the detailed development of techniques for assessing the availability of technology for operation and management in emergency situations, which will increase the accuracy of the proposed method and its introduction into the practice of machine-building enterprises.

\section{References}

1. S.P. Mitrofanov, Group technology of machinebuilding production (Mechanical Engineering, Leningrad, 1983)

2. V. Vinod, R. Sridharan, Scheduling a dynamic job shop production system with sequence-dependent setups: An experimental study Robotics and computer-integrated manufacturing $\mathbf{2 4}, 3$, pp. 435449 (2008)

3. Mohammad M. Hamasha, Azmi Alazzam, Sa'd Hamasha,Multimachine, Multimachine Flexible Manufacturing Cell Analysis Using a Markov ChainBased Approach, IEEE transactions on components packaging and manufacturing technology, 53 , pp. 439-446 (2015)

4. M. Savsar, Majid Aldaihani, A Stochastic Model for Analysis of Manufacturing Modules, Applied mathematics \& infor-mation sciences 6, 3, pp. 587600 (2012)

5. Yue Wu, A stochastic model for production loading in a global apparel manufacturing company under uncertain-ty, Production planning \& control, 22, 3, pp. 269-2812, (2011)

6. S.V. Mironov, A.M. Pishchukhin, Metasystem approach in management (Publishing house: Orenburg State. Univ., Orenburg, 2005)
7. J. Klir, Systemology. Automation of the solution of system problems (Radio and Communication, Moscow, 1990)

8. A.M. Pishukhin, Increasing the survivability of FMS based on metasystem replacements, Izvestiya Samara Scientific Center of the Russian Academy of Sciences, 18, 4-1, pp. 134-138. (2016)

9. S.V. Mironov, A.M. Pischukhin, Metasystem approach to the examination of hazardous production facilities, Successes of modern natural science 6, p. 81-82, (2005)

10. A.M. Pishchukhin, T.A. Pishchukhina, The Control Simulation of the Enterprise on the Basis Metasystem Approach, Universal Journal of Control and Automation 1, 4, p. 98-102 (2013)

11. Takahiro Kono, Keiji Ogawa, Toshiki Hirogaki, Autonomous distributed agv system based on taxi transportation strategy: effect of multiple-load agvs on conveyance efficiency, Proceedings of the asme international manufacturing science and engineering conference 2, , pp. 553-560 (2010)

12. Mohammed Ali, Subhash Wadhwa, The effect of routing flexibility on a flexible system of integrated manufacturing, International journal of production research 48,19, pp. 5691-5709 (2010)

13. Yu. A. Ivashkin, Multiagent modeling in the Simplex3 simulation system (Laboratory of Knowledge, Moscow, 2016)

14. G.F. Akhmedyanova, O.S. Eroshenko, A.M. Pishukhin, An agent-oriented approach to the modeling of the learning process, Fundamental Research, 11 (part 3) (2013)

15. A.M Pishchukhin, G.F. Akhmedyanova, Methodological support of agent-oriented modeling of the learning process, The University Complex as a Regional Center for Education, Science and Culture Materials of the All-Russian Scientific and Methodological Conference, p. 3071-3074. (2014)

16. Y. Shoham, Agent Oriented Programming, Journal of Artificial Intelligence 60 (1), pp. 51-92 (1993) 